\title{
A Wavelength Sharing and Assignment Heuristic to Minimize the Number of Wavelength Converters in Resilient WDM Networks
}

\author{
Shreejith Billenahalli*, Miguel Razo*, Wanjun Huang*, Arularasi Sivasankaran*, Limin Tang*, Hars Vardhan*, \\ Paolo Monti ${ }^{\dagger}$, Marco Tacca*, and Andrea Fumagalli* \\ * OpNeAR Lab, Erik Jonsson School of Engineering and Computer Science \\ The University of Texas at Dallas, Richardson, TX, USA \\ \{sxb071100, mrazora, wxh063000, axs075200, 1xt064000, hxv071000, mtacca, andreaf $@ @$ utdallas.edu \\ $\dagger$ NEGONET Group, School of Information and Communication Technology, ICT-FMI \\ The Royal Institute of Technology, Kista, Sweden \\ pmonti@kth.se
}

\begin{abstract}
With the successful introduction of reconfigurable optical add-drop multiplexers (ROADMs) and related technologies, WDM networks are now growing in the number of optical nodes, wavelengths, and lambda services supported. In addition, shared path protection mechanisms - whereby lambda services are allowed to share protection wavelength channels - are possible at the optical (WDM) layer. Efficient strategies must be devised to both determine the set of services that must share a common protection wavelength channel and assign wavelengths to every service. One objective of these strategies is to minimize the total number of wavelength converters (WCs), which are required every time the wavelength continuity constraint cannot be met.

This paper presents a scalable and efficient heuristic, whose goal is to minimize the number of WCs in resilient WDM networks supporting static sets of shared path protection lambda services. The heuristic comprises a set of polynomial algorithms that are executed sequentially to obtain a sub-optimal solution. In small size instances of the problem, the heuristic is compared against the optimal solution obtained from ILP formulation. For large size instances - tens of thousands of lambda services and hundreds of nodes - the heuristic yields an average number of WCs that is close to be linear in the number of services, despite the fact that the wavelength sharing factor increases.
\end{abstract}

\section{INTRODUCTION}

End-to-end optical circuits can be established in optical transport networks to support lambda services without requiring electronic processing of the service traffic at the intermediate optical cross-connect nodes. In WDM networks, the optical circuit is obtained by reserving a wavelength channel from the source to the destination node, to constitute the working path. If the lambda service requires path protection switching, one additional wavelength channel is reserved to obtain a second end-to-end optical circuit - the protection path. In some solutions the protection path is activated only if the working path fails. Depending on the lambda service requirements, the protection path may be edge, node, or shared risk link group (SRLG) disjoint from the working path.

When fiber wavelengths are a scarce resource, protection wavelength channel must be shared among as many protection paths, as long as no more than one of these protection paths is activated at any given time ${ }^{1}$. In addition, wavelength continuity is preferred when assigning wavelengths to optical circuits due to the relative high cost of wavelength converters (WCs). When the wavelength continuity constraint cannot be satisfied, WCs are necessary along the path, to shift the optical signal from the wavelength reserved in one fiber to a different wavelength reserved in the next fiber.

The problem of assigning wavelengths (WA) to optical circuits (paths) in some optimal way has been long studied. The min-RWA (optimal way of routing and wavelength assignment) is NP-hard [1]. The WA problem alone is equivalent to the coloring problem, under the assumption that WCs are not used [2], [3]. Solutions are available to sub-optimally solve the RWA problem for unprotected lambda services, in order to minimize a given cost function [4], [5]. This cost function can be defined to minimize the number of necessary WCs for each lambda service, given the set of unreserved wavelengths. For (shared path) protected lambda services, algorithms designed to minimize the number of reserved wavelengths are available for different type of traffic and protection constraints [6], [7]. A number of additional papers addresses the problem of minimizing the blocking probability of dynamically created optical circuits, for a given set of existing WCs in the network [8]. A number of more recent solutions generalize both WA and RWA problems to account for optical transmission impairments [9], [10], [11].

With the successful advent of reconfigurable optical adddrop multiplexers (ROADMs) and related technologies, WDM networks are growing in the number of optical nodes, wavelengths, and lambda services supported. Efficient strategies must be devised to both determine the set of services that must share a common protection wavelength channel and assign wavelengths to every service. One objective of these

\footnotetext{
${ }^{1}$ In this paper it is assumed that the network can be affected by at most one single failure at a time.
} 
strategies is to minimize the total number of WCs, which are required when the wavelength continuity constraint cannot be met. This problem has not been addressed in the literature. For example, some solutions apply to unprotected services only and make use of an auxiliary graph whose number of vertices is proportional to the product between the number of network nodes and wavelengths [4], [12], [13]. Other solutions target the optimization of protected lambda services but do not address the problem of WC minimization [6], [7].

This paper presents an efficient heuristic, whose goal is to minimize the number of WCs in resilient WDM networks supporting static sets of shared path protection lambda services. The heuristic comprises a set of polynomial algorithms that are executed sequentially to obtain a sub-optimal solution. In the former part of the heuristic, protection paths are assigned to shared wavelength channels while aiming for a contained number of WCs that may result from the assignment. In the latter part of the heuristic, wavelengths are assigned to both services and protection wavelength channels in order to minimize the number of required WCs. The proposed solution is referred to as the wavelength sharing and assignment (WSA) heuristic.

The polynomial algorithms in the WSA heuristic are designed to be run-time and memory efficient, and applicable to large size instances of the problem. They are successfully applied to networks with 750 nodes and above, 40 wavelengths and above, 20,000 lambda services and above, requiring only $1 \mathrm{~GB}$ of memory. The optimality of the overall solution computed by the algorithms is however difficult to assess, as this problem has not been addressed before and benchmark solutions are not available. In Section III, the WSA heuristic is compared against the solution obtained with an ILP solver for small size instances of the problem. The results indicate that the number of WCs is almost linearly proportional to the number of services, and the heuristic result is on average $40 \%$ worse when compared to the optimum found by the ILP solver. In large size instances of the problem the wavelength sharing factor - the average number of protection paths that share one wavelength channel — increases, as more options exist that may lead to wavelength sharing. Despite the fact that an increased sharing factor exacerbates the wavelength continuity constraint, the number of WCs computed by the WSA heuristic remains close to linear in the number of services.

\section{WSA HEURISTIC}

Let the optical transport network be modeled as an undirected graph $G(N, E)$, where $N$ is the set of network nodes, and $E$ is the set of edges. Each edge represents a pair of fibers, one for each direction of propagation. Let each fiber carry up to $W$ wavelengths. Let the set of lambda services contain a number of bidirectional (shared path) protected services. Assume that every protected service requires two disjoint paths (edge, node, or SRLG), i.e., a working path and a protection path, to tolerate one single network element failure. Assume that protection switching is achieved at the optical layer, i.e., the signal is generated by the transmitter (laser) and optically switched to either the working or protection path. The reverse procedure is used at the receiver, to collect the received signal from either the working or the protection path. Assume that the transmitter and receiver are both non-tunable. Then the wavelength assigned to both the working and the protection path must be the same, i.e., wavelength continuity constraint, unless wavelength conversion is allowed. Assume that both the working and protection paths for every service are given. For each conduit (fiber pair or edge) the information of all the working and protection paths which pass through it, is given.

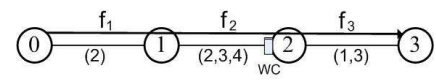

Fig. 1. Wavelength conversion along a path.

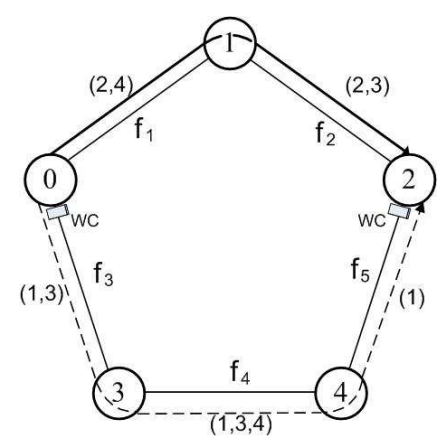

Fig. 2. Wavelength conversion at source/destination of a protected service.

The set of algorithms (WSA) described in this section aims to determine (1) which services share protection resources with one another (to form a Group of Sharing or GS), and (2) which wavelength is assigned to each service (WA), subject to minimizing the number of wavelength converters that are required in the network to support the services. Wavelength converters may be necessary for two reasons. First, due to the finite value of $W$, for some services, it may not be possible to assign a single wavelength all across to both its working and protection path due to the finite number of wavelengths (FNW) problem [4]. The FNW problem is illustrated in Fig. 1 and Fig. 2. In Fig. 1, a single wavelength from node 0 to node 3 cannot be found (the wavelengths available in each edge are in brackets). In Fig. 2, a single wavelength from node 0 to node 2 is available for either the working or protection lambda services, but as they use different wavelengths, wavelength converters are required at the source and destination. Second, services that are allowed to share protection wavelengths i.e., services in the same GS - may require more than one wavelength due to the working-protection interference (WPI) problem. The WPI problem is illustrated in Fig. 3. Two services have disjoint working paths $\left(W_{1}\right.$ and $\left.W_{2}\right)$ and are allowed to share the same protection wavelength over edges $(0,4)$ and $(1,5)$. However, protection path $P_{2}$ and working path $W_{1}$ both contain edge $(0,1)$, thus each service must be assigned 
a distinct wavelength on that edge. The protection wavelength channels on edges $(0,4)$ and $(1,5)$ are said to be undecided, as their chosen wavelength may match the wavelength of either $W_{1}$ or $P_{2}$. While it is possible to avoid the WPI problem by not allowing the two services to share the same wavelength on edges $(0,4)$ and $(1,5)$, the objective of this paper is to allow this type of sharing to minimize the total number of reserved wavelengths in the network.

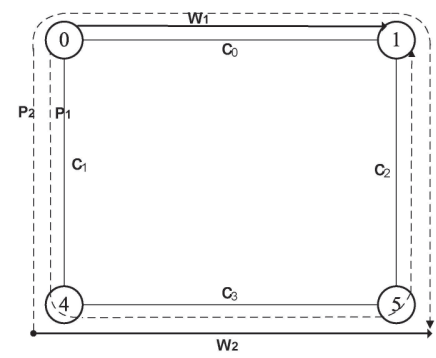

Fig. 3. Building graph for wavelength assignment.

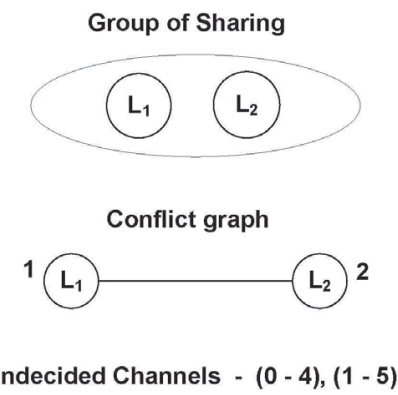

Fig. 4. Building graph for wavelength assignment.

Before presenting the set of WSA algorithms, one last observation is in order. The study in this paper makes the assumption that the protection mechanism for every service must not be sensitive to the actual location of the fault. The example in Fig. 5 illustrates the implication of this assumption using three services, each requiring edge disjoint protection. On edge $(4,5)$, one could reserve only 2 protection wavelengths to service all three services, as any single edge failure can disrupt at most 2 services. However, if only 2 protection wavelengths were reserved on edge $(4,5)$, at least one of the services would be required to use either wavelengths. For example, $P_{2}$ is assigned $\lambda_{1}$ and $P_{3}$ is assigned $\lambda_{2}$. Then $P_{1}$ is assigned either $\lambda_{1}$ if edge $(1,3)$ is faulty, or $\lambda_{2}$ if edge $(1,2)$ is faulty. To avoid this problem, three protection wavelengths must be reserved on edge $(4,5)$.

The WSA set contains a number of sequential algorithms, that are used to break the overall problem into smaller subproblems, which can be solved individually requiring less computation. This "divide and conquer" approach does not guarantee optimality. In addition, the solutions proposed for the subproblems may not be optimal, as most of the subproblems are still NP. The subproblems are: (1) to compute the

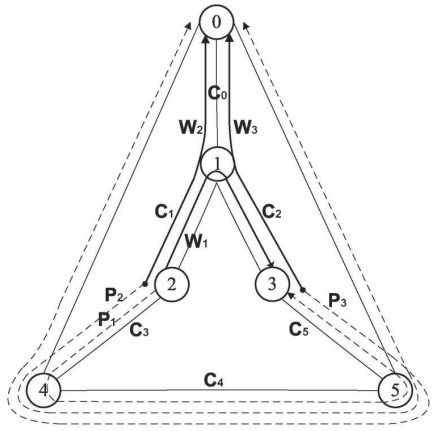

Fig. 5. Computing sharing and number of channels required.

number of shared wavelengths to be reserved on each edge (fiber) and, compute the set of services that must share the same wavelength channel on a single edge [Algorithm 1, 2 ], (2) to compute the GS (set of services that must share some protection wavelengths) [Algorithm 3, 4], (3) to assign a single lambda to as many GSs as possible [Algorithm 5], (4) to assign two or more lambdas to the remaining GSs [Algorithm 6]. Subproblem (1) is solved using a solution similar to the one described in [14]. The other steps of the proposed heuristic are not presented in [14].

\section{A. Compute Number of Shared Wavelengths}

The protection paths of multiple services may share one channel if the working paths of the corresponding services do not share any edge ${ }^{2}$. A simple data structure is associated to each edge as follows.

One edge is considered at a time. Sharing information consists of a matrix $M(i, j)$, where $i$ represents the edges that are part of the working path of the lambda services whose protection path is in the edge being considered and $j$ represents all the lambda services whose working path consists of the edge $i$. Each edge in the sharing information has the list of all such lambda services.

To determine sharing information in every edge, consider each of the protection lambda services through it. Get the working path of protection lambda service. For each edge $i$ of the working path, add the lambda service $j$ to the sharing information $M(i, j)$. Each edge in the sharing information will contain all the lambda services that have the protection path in the edge being considered.

Every shared wavelength channel must be associated with one or more services using the sharing information $M(i, j)$. For each edge in $G$, only the services whose protection path contains this edge are considered. An auxiliary graph of such services is created by representing each sharing lambda service as a node. An edge is added between the nodes of the auxiliary graph if the services are present in the sharing information $M(i, j)$ for each edge, i.e., if the working path of the lambda services are present in the same edge in the original topology graph. Then the nodes of the auxiliary graph are colored.

\footnotetext{
${ }^{2}$ Node and SRLG case are handled similarly.
} 
The node is assigned a color among all the colors which satisfies the coloring condition by considering 2 factors in order. First, a color which minimizes the WPI. Second, a color which maximizes the number of edges shared between the lambda services. The former has the highest priority and the latter factor is used only if there is no WPI. Nodes with same color represent a set of services that share the same protection channel.

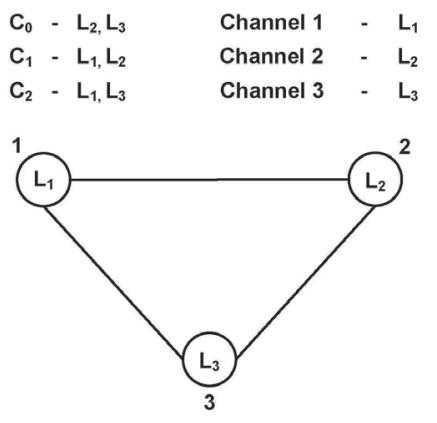

Fig. 6. Graph for the determination of sharing.

The example in Fig. 5 is used to build the auxiliary graph for determining sharing. The protection lambda services are represented as nodes as shown in Fig. 6 and the nodes are colored with distinct color since there is a conflict between every pair of nodes, hence correctly requires 3 protection channels.

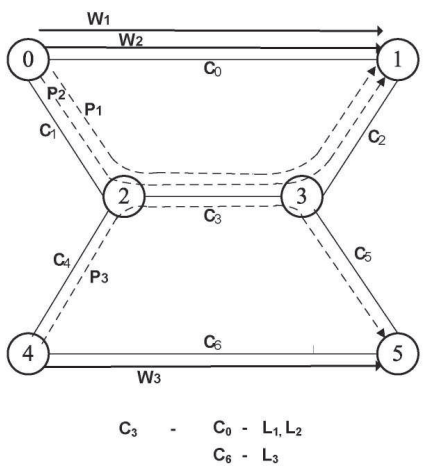

Fig. 7. Building sharing information.

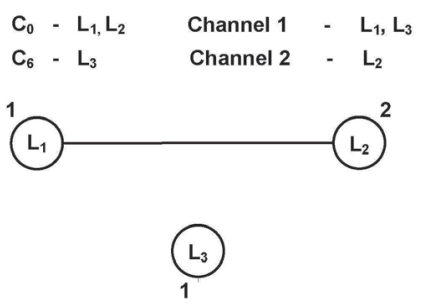

Fig. 8. Building shared channels from sharing information.

Fig. 7 shows 3 lambda service requests in a network. $L_{1}$ and $L_{2}$ are from 0 to 1 . The working path takes the route $0-1$ and the protection path takes the route $0-2-3-1 . L_{3}$ is from 4 to 5 . The working path takes the route $4-5$ and the protection path take the route $4-2-3-5$. When the sharing is computed in $C_{3}, C_{0}$ has the working paths of $L_{1}$ and $L_{2}$ and $C_{6}$ has the working paths of $L_{3}$. Fig. 8 shows the conflict graph obtained by using this information. Lambda services $L_{1}$ and $L_{3}$ are assigned color 1 and $L_{2}$ is assigned color 2 . Hence we need 2 channels one for $L_{1}$ and $L_{3}$, and the other for $L_{2}$.

Pseudo code given in Algorithm 1 and 2 describes building shared channels in an edge. An auxiliary graph, SharingGraph is created with each node representing the protection lambda service in the edge.

The node also contains the information about neighbors list and the color assigned to the node and this information is not assigned initially. Each edge $i$ in $M(i, j)$ is considered. An edge is added between the nodes for every pair of lambda services in the edge and the neighbor list of the nodes is updated.

The edge will now have the information of the number of channels required, which is the sum of the shared channels required (as determined above) and the number of working lambda services through the edge.

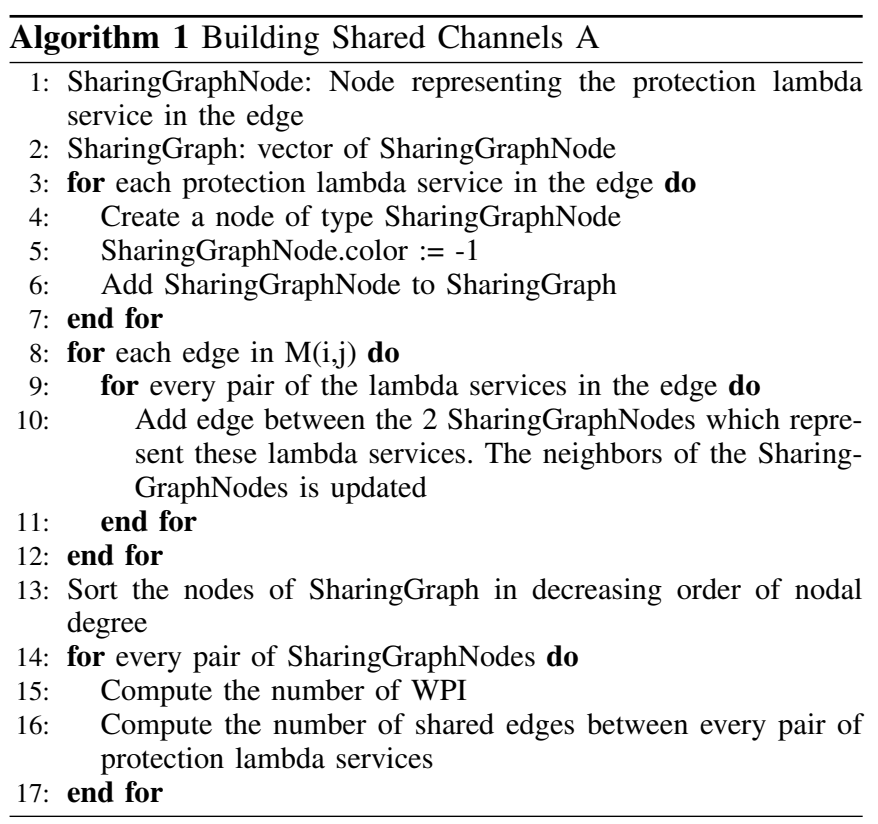

\section{B. Compute GS}

All the shared protected services which share some channel have to be grouped together, since all of these have to be assigned a common wavelength. This collection of shared protected services is called Group of Sharing $(G S)$. GS is built by scanning through the edges. All the services which are in the same channel of the edge will form the initial group. If any of the services is part of a group already formed, the group being considered will be merged with the existing group. Thus $G S$ is formed by globally considering all the edges. Due to the WPI problem, the services that belong to the same $G S$ 


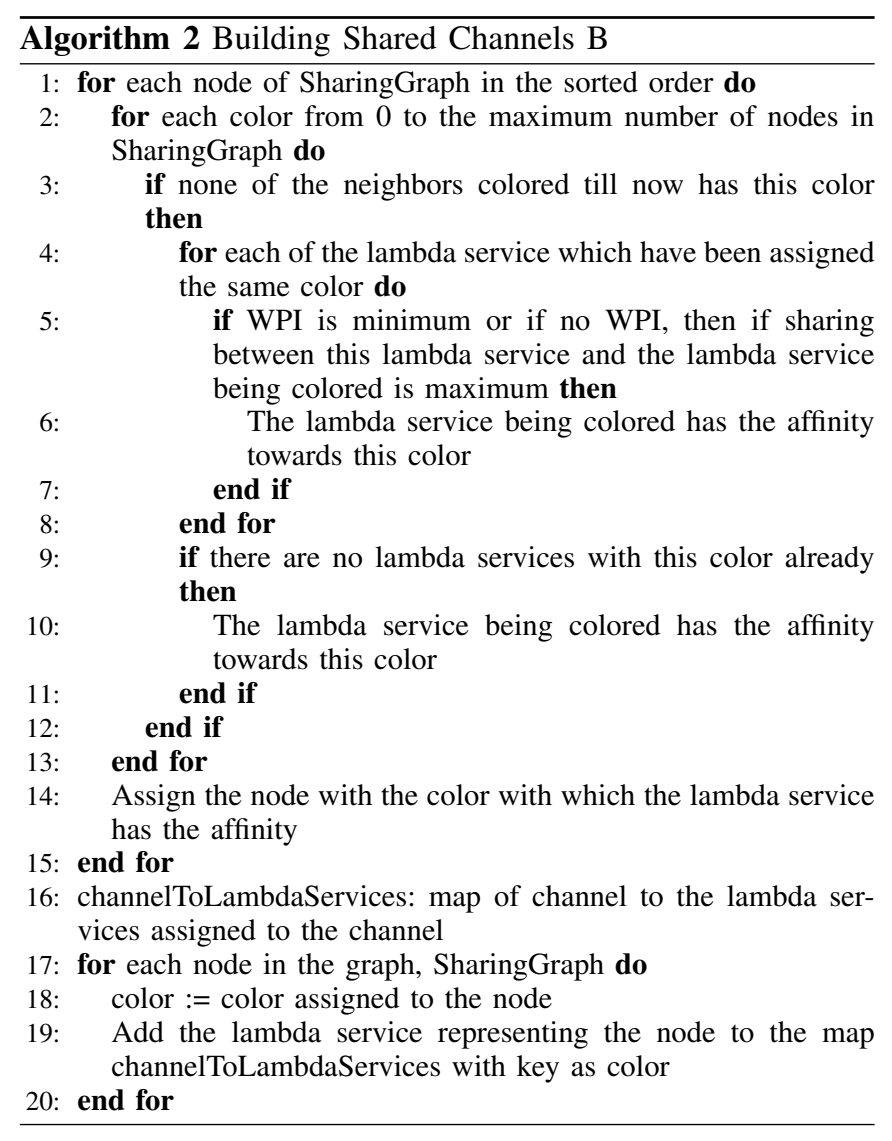

may not be assigned one single wavelength. In this case, $G S$ is partitioned to make sure that each partition does not face the WPI problem. Partitioning of $G S$ is done by coloring as follows. Each lambda service in $G S$ is represented as a node in an auxiliary graph. An edge is added between two nodes if (a) both lambda service working paths contain a common edge in $G$, or (b) the working path of one service and the protection path of the other service contain a common edge in $G$. The edge indicates that the two lambda services must use different wavelengths on at least one fiber. The auxiliary graph is colored, and the lambda services with the same color form a sub-group (partition) of $G S$. For each sub-group, a node is created in the coloring graph for wavelength assignment. As explained in Figs. 3 and 4 some of the shared channels may support lambda services that belong to two or more subgroups. These channels are termed undecided channels.

The undecided channel is assigned to the partition which has fewer number of edges of graph $G$ and the channel is removed from all other partitions. The color assigned to the partition (to which undecided channel was assigned) is assigned to the undecided channel and the same color is used by the lambda services which are in the other partitions and wavelength converters are required at both the ends of the undecided channel. Pseudo code in Algorithm 3 and 4 describes building the graph for wavelength assignment.

After the nodes for the coloring graph are determined, the edges are determined in the following way. An edge is
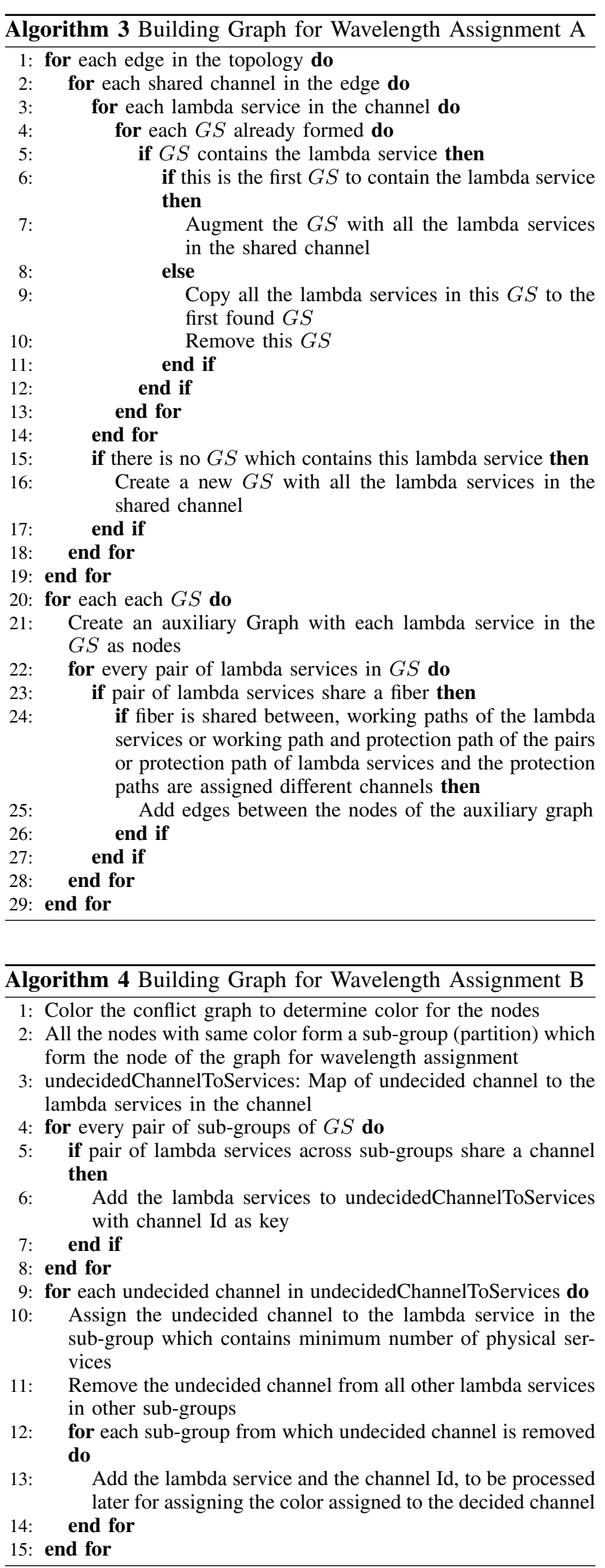
added between two nodes of the conflict graph if the nodes share at least one edge in $G$. Each node in the coloring graph contains all the edges of the lambda services which are grouped together to form a node in the coloring graph.

The WA problem is formulated as follows: Assign each service one or more dedicated wavelength values along its path(s), in order to minimize the total number of WCs, which are required in the network. This is done in two steps as described in sections II-C and II-D.

\section{Assign Single Wavelength to GS}

In the first step, wavelengths are assigned to all the services, which can be assigned a single wavelength value. This is done by coloring a conflict graph with a technique similar to the one proposed in [2]. Each lambda service is associated with a node in the conflict graph and each node represents a $G S$.

Once the conflict graph is built, the coloring is computed using any polynomial graph coloring algorithm. For example, the nodes are ordered in decreasing order of their nodal degree in the conflict graph. Each node is examined in this order and is assigned the color with the lowest identifier, which is not already assigned to any of its neighbors. The number of required colors is found at the end of this procedure. If no more than $W$ colors are required, no further steps are necessary, as every node is colored with an identifier that is within the $W$ wavelengths. If more than $W$ colors are required, colors are sorted by decreasing number of channels used by all the nodes which are assigned the color. The maximum number of nodes being assigned the color is used to break the tie. The nodes colored with any of the top ranked $W$ colors are permanently colored with the rank of the color. The other nodes are left uncolored. The color of the node is then turned into the corresponding wavelength, which is assigned to the service corresponding to the node. The colored services meet the wavelength continuity constraint, and do not require any WCs. Nodes (services) left uncolored require some WCs and are dealt with in step 2. A pseudo code description is given in Algorithm 5.

\section{Assign Multiple Wavelengths to GS}

For every $G S$ left uncolored after running step 1, the following algorithm is used to assign two or more wavelength values, i.e., wavelength converter, to the services in the $G S$. Consider one uncolored $G S$ at a time. Create a subgraph of $G$, which is formed by only the edges and the nodes that belong to the service paths in the uncolored $G S$. Sort the edges by increasing number of unreserved wavelengths. Sort the wavelength values by decreasing wavelength popularity, i.e., the popularity of a wavelength is defined as the number of edges in the subgraph of $G$, which have that wavelength value unreserved. Every edge in the subgraph is uncolored and it is then colored using one of its unreserved wavelengths as follows. Start with the uncolored edge, which has the smallest number of unreserved wavelengths. Color that edge with the most popular wavelength value that is available on that edge. Let this wavelength value be the temporary default wavelength.

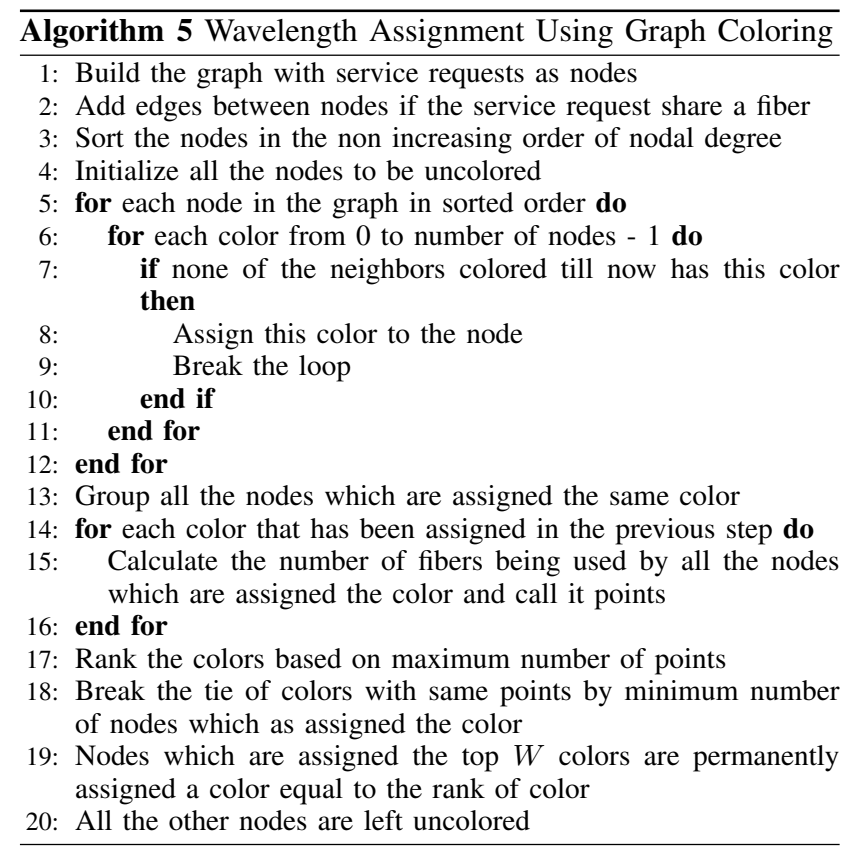

This colored edge forms a fragment of the subgraph of $G$. The fragment is augmented by adding only edges which can be colored using the default wavelength, as follows. Every edge which is adjacent to the fragment is added to the fragment if the default wavelength is unreserved on the edge. Every edge added to the fragment is colored with the default wavelength. The algorithm keeps adding edges to the fragment till no additional edge can be added. The following iterative steps are then performed until all edges in the subgraph of $G$ are colored. Create a new fragment with the uncolored edge, which has the smallest number of unreserved wavelengths. Color that edge with the most popular wavelength value that is available on that edge. This wavelength value is the new default wavelength, and this fragment is augmented as described earlier, till no more edges can be added. A pseudo code description of step 2 is given in Algorithm 6.

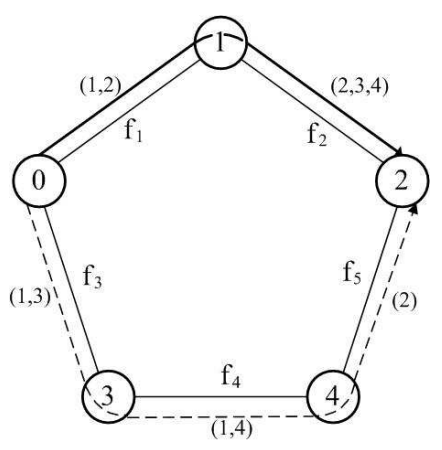

Fig. 9. Step 2: Example using a protected lambda service.

The execution of step 2 is illustrated with the help of Fig. 9, which shows the subgraph of $G$ obtained for a protection 


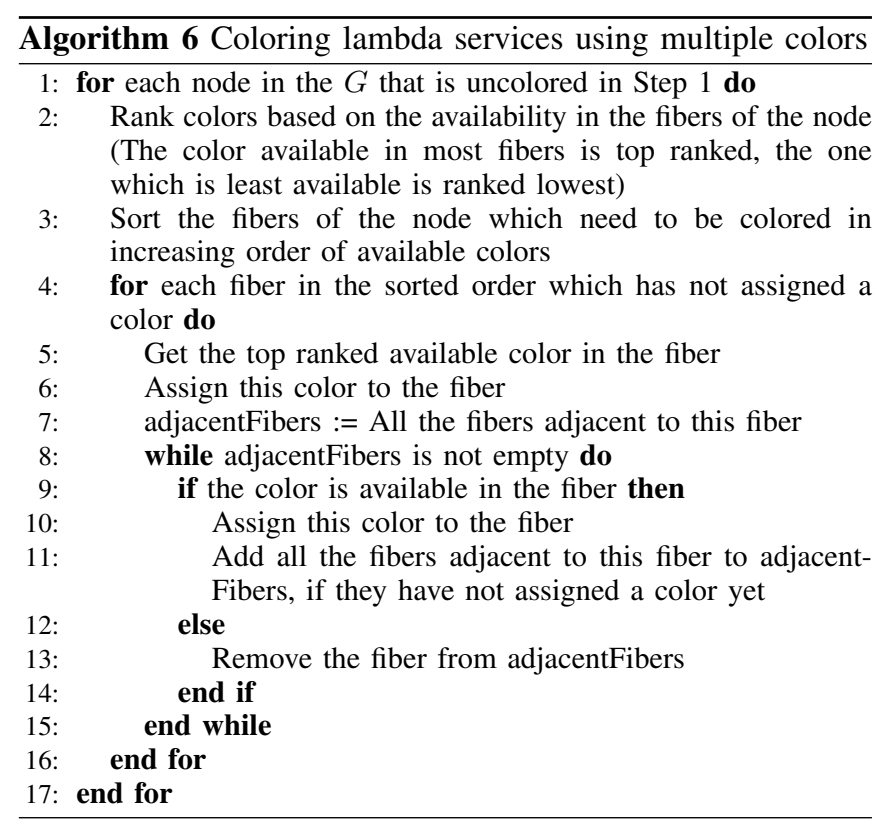

lambda service. The protection service has source node 0 and destination node 2 . The working path (solid arrow) takes route $0-1-2$ and the protection path (dashed arrow) takes route 0-3-4-2. The unreserved wavelengths (colors) on each edge of the subgraph are shown in brackets, next to the edge. The wavelength continuity constraint cannot be met for this service, and step 2 is therefore required. $f_{1}, f_{2}, f_{3}, f_{4}$ and $f_{5}$ represent the fibers in the corresponding edges. The algorithm first sorts the wavelengths based on their decreasing popularity. Here wavelength 1 is available in 3 fibers, 2 is available in 3 fibers, and so on. The wavelengths are then sorted as 1, 2, 3 and 4 . The edges (fibers) are sorted with increasing number of unreserved wavelengths: $f_{1}$ has 2 unreserved wavelengths, $f_{2}$ has 3 , and so on. Edges are thus sorted as follows: $f_{5}$, $f_{1}, f_{3}, f_{4}$ and $f_{2} . f_{5}$ is considered first and is assigned the only unreserved wavelength, i.e., 2. $f_{5}$ constitutes the first fragment. All edges which are adjacent to the fragment $\left(f_{2}\right.$ and $f_{4}$ ) are considered next. $f_{2}$ can be added to the fragment as it can be assigned wavelength 2 , too. $f_{4}$ cannot be added to the fragment, as wavelength 2 is not unreserved on that edge. The fragment now contains both $f_{5}$ and $f_{2}$, and $f_{1}$ is an adjacent edge to the fragment. $f_{1}$ is added to the fragment and assigned wavelength 2 . At this point the fragment cannot be further augmented. The algorithm then creates a new fragment by choosing the uncolored edge with the smallest number of unreserved wavelengths. In this case, both $f_{3}$ and $f_{4}$ have each 2 unreserved wavelengths. Let $f_{3}$ be the chosen edge. $f_{3}$ is assigned wavelength 1 , which is more popular than wavelength 3 . The new fragment is augmented by adding $f_{4}$, which is assigned wavelength 1 , too. All the edges in the subgraph are now colored and the algorithm stops. In summary, this lambda service is assigned two wavelength values: wavelength 2 over edges $f_{1}, f_{2}$ and $f_{5}$ and wavelength 1 over edges $f_{3}$ and $f_{4}$. Two bidirectional WCs are then required: one at node 4 , along the protection path; the other at node $0^{3}$.

After assigning colors to all the nodes, the undecided channels which were formed while building the graph for coloring need to be handled. All the undecided channels which were removed before coloring are added to the lambda services and are assigned the color assigned to the retained channel which was decided to go with one node. Pseudo code in Algorithm 7 describes the handling of undecided channels.

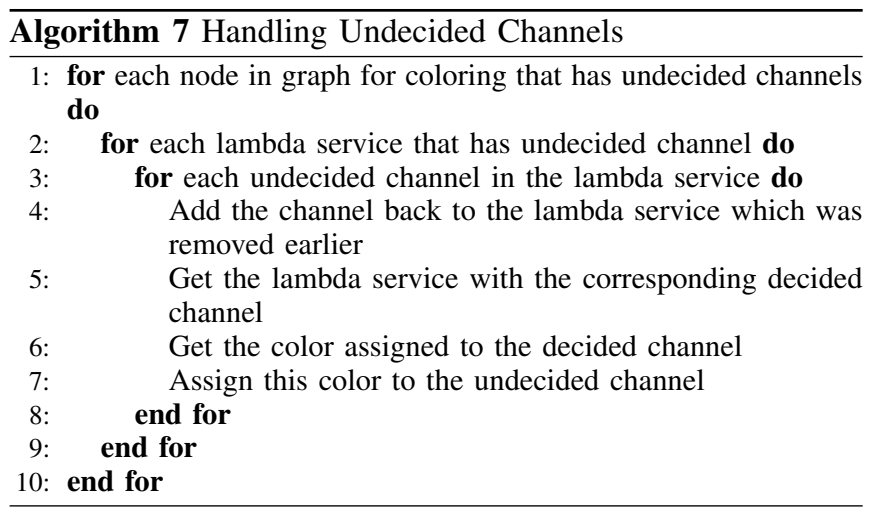

\section{RESULTS}

In this section, both the optimality and scalability of the WSA heuristic are assessed.

Benchmarking. The WSA heuristic optimality is investigated first. As already mentioned, no other solution is available in the literature that can be used as benchmark. An ILP formulation [15] is therefore used for small instances of the problem. A network with a number of nodes $N=5$, a number of fiber links (edges) $L=8$ and a number of wavelengths per fiber $W=8$ is used. The number of protected services ${ }^{4}$ is varied from 5 to 12. For each number of lambda services, 20 experiments are run. The results are averaged. For each experiment, the ILP formulation and the WSA heuristic are solved using the same set of input lambda services where, for each service the same routing is used. Results in TABLE I show the comparison. The average number of required channels is the same for both the ILP and the WSA heuristic. The table also shows the average number of required WCs. The WSA heuristic requires a number of WCs that is $27 \%$ to $48 \%$ larger than the optimal number obtained with the ILP.

Scalability. The scalability of the WSA heuristic is assessed next. TABLE II shows results obtained by the WSA heuristic when the network size - i.e., the number of nodes, fiber links (edges), and lambda services - is increased. The number of wavelengths per fiber is set to $W=40$. The table shows the number of nodes, fiber links, WCs required, sharing factor (i.e., average number of services sharing one protection

\footnotetext{
${ }^{3}$ The transmitter at node 0 can be assigned wavelength 2 (1), and the WC is placed between the transmitter and the multiplexer of $f_{3}\left(f_{1}\right)$

${ }^{4}$ The ILP solver [16] requires several hours to compute the optimal solution for the ILP formulation when the number of lambda services is larger than 12.
} 
TABLE I

ILP VS. WSA HEURISTIC.

\begin{tabular}{|c|c|c|c|c|}
\hline & \multicolumn{2}{|c|}{ ILP } & \multicolumn{2}{c|}{ WSA } \\
\hline Req. & WC & Channels & WC & Channels \\
\hline \hline 5 & 1.3 & 14.1 & 1.65 & 14.1 \\
\hline 6 & 1.75 & 16.6 & 2.25 & 16.6 \\
\hline 7 & 2.35 & 18.9 & 3.45 & 18.9 \\
\hline 8 & 3.15 & 20.85 & 4.65 & 20.85 \\
\hline 9 & 4.05 & 22.85 & 5.7 & 22.85 \\
\hline 10 & 4.85 & 24.95 & 6.8 & 24.95 \\
\hline 11 & 5.8 & 27.25 & 8.3 & 27.25 \\
\hline 12 & 6.65 & 29.45 & 9.4 & 29.45 \\
\hline \hline
\end{tabular}

channel), wavelength channels used, and the time (in seconds) taken by the WSA heuristic, respectively. The run time is reasonable, even for large networks with hundreds of nodes and tens of thousands of lambda services.

TABLE II

SCALABILITY OF THE WSA HEURISTIC.

\begin{tabular}{|l|l|l|l|l|l|l|}
\hline $\mathrm{N}$ & $\mathrm{F}$ & Req. & WC & Sharing & Channels & Time [s] \\
\hline \hline 30 & 133 & 2194 & 8361 & 5.02763 & 9516 & 78 \\
\hline 40 & 179 & 2499 & 10125 & 5.34633 & 11560 & 104 \\
\hline 50 & 223 & 2995 & 12671 & 5.94234 & 14484 & 157 \\
\hline 75 & 335 & 3500 & 15318 & 6.5274 & 18160 & 201 \\
\hline 100 & 446 & 5974 & 29839 & 7.63172 & 32406 & 674 \\
\hline 150 & 666 & 7989 & 41556 & 8.08658 & 46740 & 1245 \\
\hline 200 & 890 & 9998 & 53918 & 8.59914 & 61034 & 2052 \\
\hline 300 & 1337 & 14927 & 86273 & 9.40011 & 96536 & 5215 \\
\hline 400 & 1789 & 17994 & 104883 & 9.64224 & 121598 & 7729 \\
\hline 500 & 2231 & 21985 & 131515 & 9.92145 & 154760 & 12492 \\
\hline 750 & 3351 & 26000 & 157735 & 9.66434 & 195634 & 18938 \\
\hline \hline
\end{tabular}

\section{SUMMARY}

This paper presented the polynomial WSA heuristic, whose goal is to minimize the number of WCs in resilient WDM networks supporting lambda services protected with shared path protection. The WSA heuristic $(i)$ computes the set of protection paths that can share the same wavelength channel, and $(i i)$ assigns wavelengths to both services and protection wavelength channels, given a pre-computed set of routes for every service's working and protection path.

The WSA heuristic is designed to be time and memory efficient, and applicable to large size instances of the problem. It is successfully applied to networks with 750 nodes and above, 40 wavelengths and above, 20,000 lambda services and above, requiring only $1 \mathrm{~GB}$ of memory. The optimality of the overall solution computed by the algorithms is however difficult to assess. A comparison with an ILP formulation was carried out in small size problem instances. In large size instances of the problem, where the wavelength sharing factor increases and the wavelength continuity constraint is exacerbated, the number of WCs computed by the WSA heuristic is close to linear with the number of services.

\section{REFERENCES}

[1] T. Erlebach, K. Jansen, and C. Elvezia, "The complexity of path coloring and call scheduling," Theoretical Computer Science, vol. 255, p. 2001, 2001.
[2] I. Chlamtac, A. Ganz, and G. Karmi, "Lightpath communications: An approach to high bandwidth optical wans," IEEE Transaction on Communications, vol. 40, no. 7, pp. 1171-1182, July 1992.

[3] G. Li and R. Simha, "The partition coloring problem and its application to wavelength routing and assignment," in Proceedings of the First Workshop on Optical Networks, 2000.

[4] I. Chlamtac, A. Farago, and T. Zhang, "Lightpath (wavelength) routing in large wdm networks," IEEE Journal on Selected Areas in Communications, vol. 14, no. 5, pp. 909-913, Jun 1996.

[5] D. Banerjee and B. Mukherjee, "A practical approach for routing and wavelength assignment in large wavelength-routed optical networks," IEEE Journal on Selected Areas in Communications, vol. 14, pp. 903908, 1996.

[6] A. Jaekel and T. Khan, "Routing and wavelength assignment in optical mesh networks with wavelength conversion," in Proceedings of 25th IEEE International Performance, Computing, and Communications Conference (IPCCC 2006), April 2006, pp. 273-280.

[7] H. Zang, C. Ou, and B. Mukherjee, "Path-protection routing and wavelength assignment (rwa) in wdm mesh networks under duct-layer constraints," IEEE/ACM Transactions on Networking, vol. 11, no. 2, pp. 248-258, Apr 2003.

[8] X. Chu, B. Li, and Z. Zhang, "A dynamic rwa algorithm in a wavelengthrouted all-optical network with wavelength converters," in In Proceedings of IEEE INFOCOM 2003, March-April 2003, pp. 1795-1804.

[9] R. Martinez, F. Cugini, N. Andriolli, L. Valcarenghi, P. Castoldi, L. Wosinska, J. Comellas, and G. Junyent, "Challenges and requirements for introducing impairment-awareness into the management and control planes of ason/gmpls wdm networks," IEEE Communications Magazine, vol. 44, no. 12, p. 76, 2006.

[10] Y. Huang, J. P. Heritage, and B. Mukherjee, "Connection provisioning with transmission impairment consideration in optical wdm networks with high-speed channels," Journal of Lightwave Technology, vol. 23, no. 3, p. 982, 2005 .

[11] A. Jirattigalachote, K. Katrinis, A. Tzanakaki, L. Wosinska, and P. Monti, "Quantifying the benefit of ber-based differentiated path provisioning in wdm optical networks," in Proceedings 11th International Conference on Transparent Optical Networks (ICTON 09), July 2009.

[12] S. Gowda and K. M. Sivalingam, "Protection mechanisms for optical wdm networks based on wavelength converter multiplexing and backup path relocation techniques," in Proceedings of IEEE INFOCOM 2003, March-April 2003.

[13] Y. Wang, L. Li, and S. Wang, "A new algorithm of design protection for wavelength-routed networks and efficient wavelength converter placement," in Proceedings of IEEE International Conference on Communications (ICC 2001), vol. 6, 2001, pp. 1807-1811.

[14] J.-F. Labourdette, E. Bouillet, R. Ramamurthy, and G. Ellinas, Path Routing in Mesh Optical Networks. John Wiley \& Sons, 2006.

[15] W. Huang et al., "Shared Protection ILP Formulation," The University of Texas at Dallas, Tech. Rep., May 2009. [Online]. Available: http://opnear.utdallas.edu/publications/reports/UTD-EE-03-2009.pdf

[16] Ilog, Inc., "Solver cplex," 2009, http://www.ilog.com/products/cplex/. [Online]. Available: http://www.ilog.com/products/cplex/ 